\title{
Ablación térmica de nervios intercostales por radiofrecuencia para dolor por fracturas costales múltiples
}

\author{
Radiofrequency ablation of intercostal nerves for \\ pain management in multiple rib fractures \\ Rocío Torres Méndez, ${ }^{*}$ Felipe Vega Rivera, ${ }^{\star}$ Rafael Acuña Prats ${ }^{\S}$
}

Palabras clave:

Dolor, ablación térmica de nervios intercostales, trauma contuso de tórax.

Keywords: Pain, intercostal nerve ablation, blunt thoracic trauma.

${ }^{*}$ Jefa de la Clínica de Dolor.

¥ Cirujano de los equipos de Trauma. Coordinador del Centro de Enseñanza e Investigación.

$\S$ Cirujano General

y Endoscopista de la Clínica de Dolor.

Hospital Ángeles Lomas. Ciudad de México, México.

Recibido: 21/02/2019 Aceptado: 05/02/2020

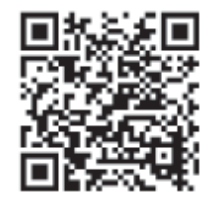

\section{RESUMEN}

Introducción: El trauma en general y el trauma contuso de tórax en particular continúan formando parte de los problemas más frecuentes que el médico, el cirujano y los sistemas de emergencia tienen que afrontar todos los días. El dolor que experimentan los pacientes suele ser muy intenso, limitando la mecánica respiratoria de manera importante y por consiguiente, complicándose el paciente con frecuencia por atelectasias pulmonares y focos neumónicos al no contar con un buen manejo de secreciones. El objetivo de este artículo es demostrar que el uso de la ablación por radiofrecuencia de los nervios intercostales disminuye el dolor en el paciente con trauma contuso de tórax, mediante la revisión de 12 casos con trauma contuso y fracturas costales múltiples tratados con radiofrecuencia guiada con fluoroscopia, ultrasonido e impedancia para producir ablación térmica de los nervios intercostales involucrados y de esta manera, reducir de manera importante el dolor preoperatorio o postoperatorio asociado a fracturas costales múltiples. Material y métodos: Tipo de estudio retrospectivo de 12 pacientes con trauma de tórax contuso y dificultad para ventilar, con una cinemática de: caídas de diferentes alturas (de 10 a 25 $\mathrm{m}$ de altura), accidentes automovilísticos, y de motocicleta. Previo consentimiento informado para este tipo de procedimiento y bajo anestesia general e intubación se realizó ablación de nervios intercostales, utilizando fluoroscopia para localizar el nervio intercostal involucrado proximal a la fractura, se corroboró la correcta posición de la aguja mediante impedancia, estimulación motora y sensitiva. Se efectuó ablación a $60{ }^{\circ} \mathrm{C}$ durante un minuto por cada nervio empleando el equipo NeuroTherm NT 1100 (marca registrada). Variables: cualitativas descriptivas: edad, sexo, cinemática del trauma. Variables en estudio: dolor a la ventilación preablación y postablación, medidas mediante escala numérica de dolor (END), dolor a la espiración preablación y postablación. Variables cuantitativas: número de costillas

\section{ABSTRACT}

Introduction: Trauma continues to be a major problem that is confronted daily by doctors, surgeons and the emergency systems in general, particularly in blunt thoracic trauma. The pain that the patients have usually is severe restricting in an important way the ventilation and furthermore it generally produces lung atelectasis and neumony because the patients can t move lung secretions. The main objective of this article is to show that the use of intercostals nerve ablation diminishes the pain of the patient with blunt thoracic trauma by the review of the first 12 cases of blunt thoracic trauma with multiples rib fractures treated with radiofrequency ablation of the intercostal nerves guided by fluoroscopy or ultrasound and electric impedance in order to diminish the pain before or after surgery. Material and methods: It was a retrospective study of 12 patients with blunt thoracic trauma who had difficulties with the ventilation because of the intensity of the pain. The cause of the trauma were car and motorcycle accidents, falls form different heights $(10-25 \mathrm{~m})$. The patients had general anesthesia and intubation before the procedure followed by ablation of the intercostal nerves proximal to the rib fracture guided by fluorosocopy or ultrasound and impedance. The correct placement of the needle was done by electric impedance and motor and sensory stimulations. The nerve ablation of the intercostals nerves of the rib fractures were done with $60^{\circ} \mathrm{C}$ for one minute for each nerve using the NeuroTherm NT 1100 (registered marc). Variables: qualitative variables used for description of the population was accomplished by age, gender, mechanism of the trauma. Pain intensity was measured by the quantitative pain number scale before and after the treatment and with exhalation, before and after treatment, quantitative variables studied: number of rib fractured, injured severity scale score, and revised trauma score, morphine use in $m g$ before and after the treatment description of the result

Citar como: Torres MR, Vega RF, Acuña PR. Ablación térmica de nervios intercostales por radiofrecuencia para dolor por fracturas costales múltiples. Cir Gen. 2020; 42(1): 24-30. doi: 10.35366/92708 
fracturadas, calificación del Injury Severity Score (ISS) y Revised Trauma Score (RTS). Variables cuantitativas del estudio: consumo de morfina en mg preablación y postablación. Descripción de las variables mediante: media y desviación estándar. Resultados: Disminución del dolor a la espiración en 70\%, así como del consumo de opioides en $70.8 \%$. Complicaciones: alodinia leve y pasajera en $41.2 \%$ de los casos controlada con gabapentinoides durante dos semanas. Conclusión: La ablación térmica de nervios intercostales por radiofrecuencia en trauma contuso de tórax es útil, disminuye el dolor en forma significativa y permite mejor ventilación con menor consumo de analgésicos. Esta técnica podría perfilarse como un nuevo estándar de oro en el control del dolor en trauma torácico severo.

\section{INTRODUCCIÓN}

E I trauma es un problema de salud pública a nivel mundial y una de las principales causas de mortalidad en todos los grupos etarios. El dolor en trauma con frecuencia requiere opiáceos y no es raro que se encuentre subtratado.

En particular el trauma contuso de tórax es un problema frecuente, ya sea por accidentes automovilísticos al no usar el cinturón de seguridad, motocicleta, caídas de bicicleta, o de altura y atropellamiento. Son frecuentes las fracturas costales múltiples, las cuales pueden ir acompañadas de hemoneumotórax o inclusive de tórax inestable. Independientemente del riesgo que estas patologías pueden representar para la vida del paciente, una vez que se resuelven en la evaluación inicial según el protocolo Advanced Trauma Life Support (ATLS) del Colegio Americano de Cirujanos, el dolor persiste. Un dolor por lo regular muy intenso, de nueve y 10 en la escala numérica del dolor, que se intensifica a la inspiración profunda y que limita de manera importante la mecánica ventilatoria del enfermo retardando el tiempo de extubación en las unidades de terapia intensiva debido al dolor a la inspiración y al mal manejo de secreciones al no poder expectorar.

Este dolor necesita por lo regular dosis altas de opiáceos con los efectos colaterales que esto implica como náusea, vómito, cefalea, depresión respiratoria, sedación del enfermo y delirio, pudiendo además enmascarar otras lesiones como traumatismo by average and standard deviation. Results: Pain intensity was diminished in $70 \%$, and the daily morphine consumption was also diminished in $70.8 \%$. Alodine as a complication of the treatment was found in a $41.2 \%$ of the cases and was easily controlled with gabapentinoids for two weeks. Conclusion: The use of intercostal nerve ablation with radiofrequency in thoracic blunt trauma is effective and safe. It controls the pain significantly and improves the ventilation of the patient diminishing the morphine consumption. This technique could become the new gold standard in the treatment of pain in the severe thoracic blunt trauma.

craneoencefálico, trauma contuso de abdomen y síndrome compartimental, que no es raro que coincidan las tres entidades en esta cinemática de trauma.

Desde 1945 el bloqueo de nervios torácicos se ha utilizado para disminuir el dolor por trauma torácico. Durante la Segunda Guerra Mundial fue un recurso valioso para reducir el dolor de las costillas fracturadas. ${ }^{1,2}$

Sin embargo, aunque la infiltración de anestésicos a los nervios intercostales afectados producía alivio importante del dolor y reducción de los analgésicos, era de corta duración. ${ }^{3,4}$

La instalación de un catéter peridural con infusión continua de opiáceos mejoró la analgesia de estos pacientes; sin embargo, presentaba el problema de la movilización de un enfermo politraumatizado para poder Ilevarla a cabo, además de la hipotensión asociada que podía aparecer a la infusión continua por vasodilatación asociada en pacientes hemodinámicamente inestables o con traumatismo craneoencefálico agravando el estado de choque y disminuyendo la perfusión cerebral.5,6

La radiofrecuencia actúa al producir ablación térmica del nervio intercostal involucrado mediante una aguja que se coloca en el canal subcostal guiado, ya sea por ultrasonido o fluoroscopia. ${ }^{7-10}$

Este daño permanente del nervio intercostal permite una importante analgesia, puede realizarse con el paciente en decúbito dorsal sin necesidad de cambios de posición e inclusive dentro de las unidades de terapia intensiva si 
se utiliza como guía el ultrasonido, además la analgesia es permanente, lo que permite al paciente una buena mecánica respiratoria, previene atelectasias pulmonares o neumonía y disminuye el uso de analgésicos. ${ }^{11-15}$

Con base en lo anterior se comenzó a realizar radiofrecuencia de los nervios intercostales en nuestro hospital para el tratamiento del dolor y mejorar la mecánica respiratoria en los pacientes con trauma contuso de tórax. Este trabajo surge de la necesidad de poder controlar el dolor severo que presentan los pacientes y cómo mejorar su mecánica respiratoria para una extubación temprana; y se ha perfilado como una opción al tratamiento médico y quirúrgico tradicional del paciente en nuestro hospital.

El objetivo de este artículo es describir la serie de casos tratados mediante este método en el Hospital Ángeles Lomas.

\section{MATERIAL Y MÉTODOS}

Se revisaron los expedientes físicos y, en su caso, electrónicos de manera retrospectiva de los pacientes con trauma contuso de tórax que fueron tratados con radiofrecuencia de nervios intercostales en el Hospital Ángeles Lomas de enero de 2006 a agosto de 2018. Durante este periodo se trataron con esta técnica 12 pacientes, previo consentimiento informado del paciente y/o de su familia para la técnica de ablación térmica de nervios in-

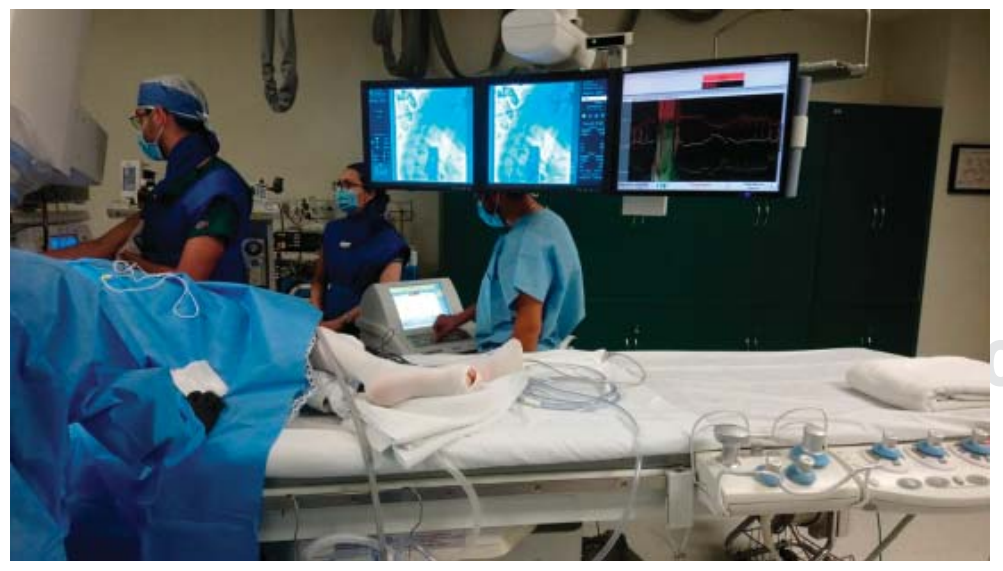

Figura 1: Control fluoroscópico de ablación por radiofrecuencia de intercostales en fracturas costales múltiples. tercostales, además de los correspondientes consentimientos informados para otros tipos de tratamiento quirúrgico que el paciente ameritara, con trauma contuso de tórax y fracturas costales múltiples.

Los pacientes fueron reanimados de acuerdo al protocolo del ATLS. Posterior a su estabilización y habiéndose tratado lesiones prioritarias de otros órganos, (de 24 a 72 horas después) los pacientes fueron sometidos a radiofrecuencia en sala de fluoroscopia, previa intubación, o mediante control ultrasonográfico en la misma unidad de terapia intensiva.

Se utilizaron agujas para termoablación marca NeuroTherm, modelo Simplicity III, marca registrada. Se localizó el nervio intercostal involucrado proximal a la fractura, se corroboró la correcta posición de la aguja mediante impedancia, estimulación motora y sensitiva y se realizó la ablación de los nervios intercostales a $60{ }^{\circ} \mathrm{C}$ durante un minuto por cada nervio utilizando el equipo NeuroTherm NT 1100 (Figura 1).

Se evaluó el dolor mediante la escala numérica del dolor. Al reposo con respiración superficial y a la espiración prerradiofrecuencia y postradiofrecuencia. De manera indirecta la intensidad del dolor también se midió a través del consumo diario de opiáceos. Todos los pacientes se encontraban conscientes al evaluar el dolor.

Para poder medir el consumo de opiáceos prerradiofrecuencia y postradiofrecuencia se documentaron los mg de morfina administrados por vía endovenosa en 24 horas que requirió cada paciente. En los casos que no se utilizó morfina se convirtieron a mg de morfina buscando la equivalencia de los otros opiáceos a morfina con objeto de poder unificar y comparar el consumo diario de morfina pretratamiento y postratamiento.

\section{RESULTADOS}

Durante este periodo se trataron con esta técnica 12 pacientes con trauma contuso de tórax y fracturas costales múltiples. Cinco pacientes femeninos y siete masculinos, con rango de edad de 28 a 76 con un promedio de 57.5 años, con la siguiente cinemática de trauma: 
seis accidentes automovilísticos, dos de moto y cuatro caídas (de su propia altura, 10 y 25 $\mathrm{m})$, con un rango de costillas fracturadas de tres a 10 y un promedio de seis. La intensidad del traumatismo se graduó mediante la escala combinada de trauma score y con el índice de severidad de las lesiones (TRISS) de la Asociación Americana de Cirugía de Trauma con un rango de 1.1 a 49.7, una media de 11.71 y una desviación estándar de 13.12 puntos. Se calificó a los pacientes con trauma contuso como severo utilizando estas escalas de 1 a 12 (8.3\%).

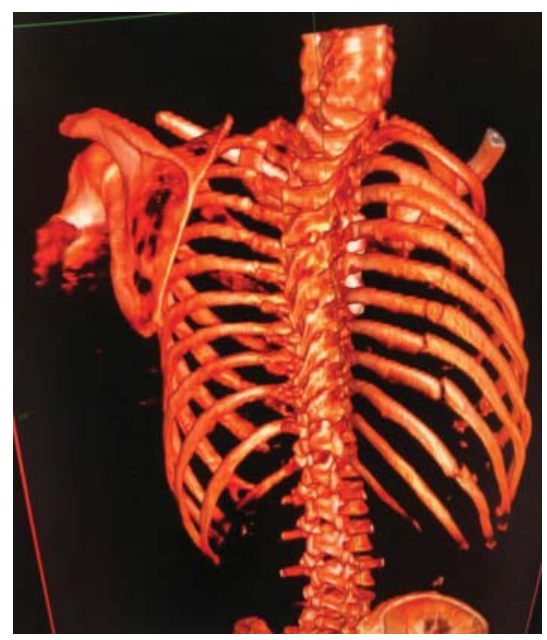

Figura 2: Fracturas costales múltiples por trauma contuso, tercera a décima costillas hemitórax derecho. Tomografia con reconstrucción ósea.
Los 12 pacientes tuvieron una media de costillas fracturadas de 5.75 con una desviación estándar de 2.18. Se midió el dolor al reposo con respiración superficial y a la espiración (Figura 2).

El dolor al reposo con respiración superficial antes de la radiofrecuencia tuvo una media de 7.6 puntos en la escala numérica con una desviación estándar de 2.18 puntos. Posterior a la ablación de los nervios intercostales la media fue de 2.08 con una desviación estándar de 1.73 en la escala numérica del dolor. El dolor a la espiración se midió antes de la radiofrecuencia con una media de 9.75 y una desviación estándar de 0.62 y posterior a la misma con una media de 2.75 y una desviación estándar de 1.36 utilizando esta escala.

La dosis en mg por día de opiáceos equivalente a morfina administrada por vía intravenosa antes de la radiofrecuencia tuvo una media de $26.67 \mathrm{mg}$ en 24 horas con una desviación estándar de $10.5 \mathrm{mg}$ y posterior a la radiofrecuencia fue de $7.79 \mathrm{mg}$ en 24 horas con una desviación estándar de 0.28 (Tabla 1).

El dolor disminuyó en 70\% postradiofrecuencia al reposo, a la espiración también en $70 \%$, y el consumo de opiáceos en mg por 24 horas se redujo en $70.8 \%$.

Las complicaciones observadas de la técnica de radiofrecuencia fueron de alodinia leve y pasajera en $41.2 \%$ de los enfermos que se controlaron con gabapentinoides cada ocho horas

Tabla 1: Evaluación de dolor postablación y consumo de morfina ( $\mathrm{N}=12)$.

\begin{tabular}{|c|c|c|c|}
\hline & Femenino & Masculino & Total \\
\hline Variable & Media \pm DE & Media \pm DE & Media \pm DE \\
\hline Número de costillas rotas & $5.4 \pm 2.70$ & $6.00 \pm 1.91$ & $5.75 \pm 2.18$ \\
\hline Dolor (EVA) reposo c/respiración superficial PreAbx DRSPA & $7.2 \pm 2.39$ & $7.71 \pm 2.36$ & $7.50 \pm 2.28$ \\
\hline Dolor (EVA) reposo c/respiración superficial PostAbx DRSPOA & $2.6 \pm 1.52$ & $1.71 \pm 1.89$ & $2.08 \pm 1.73$ \\
\hline Dolor (EVA) espiración normal PreAbx DEPA & $10.0 \pm 0.00$ & $9.57 \pm 0.79$ & $9.75 \pm 0.62$ \\
\hline Dolor (EVA) espiración normal PostAbx DEPOA & $3.0 \pm 1.22$ & $2.57 \pm 1.51$ & $2.75 \pm 1.36$ \\
\hline Dosis/día mg opioide equivalente a morfina PreAbx DO = M & $25.8 \pm 13.40$ & $27.29 \pm 12.85$ & $26.67 \pm 12.49$ \\
\hline Mín.-máx. & $10-40$ & $10-50$ & $10-50$ \\
\hline Dosis opioide $(\mathrm{mg})$ equivalente a morfina PostAbx DO = M PosA & $7.8 \pm 6.11$ & $7.79 \pm 10.30$ & $7.79 \pm 8.45$ \\
\hline Mín.-máx. & $0-16.5$ & $0-28$ & $0-28$ \\
\hline
\end{tabular}


durante dos semanas. No hubo complicaciones graves ni mortalidad en esta serie. En algunos casos se combinó la fijación de las costillas fracturadas además de la ablación térmica de los intercostales transoperatoria cuando el paciente presentaba tórax inestable o desplazamiento de las costillas.

Las complicaciones atribuidas a esta técnica fueron menores principalmente por alodinia leve y pasajera en $41.2 \%$ de los casos controlada con gabapentinoides a dosis bajas durante dos semanas. No hubo ningún caso de neumotórax postablación ni de mortalidad en esta serie.

\section{DISCUSIÓN}

El dolor es considerado en la actualidad como el quinto signo vital y el alivio del mismo ya se considera un derecho fundamental del paciente a nivel internacional, decretado por la Organización Mundial de la Salud (OMS) como uno de los derechos humanos y se incurre en una falta grave de la ética profesional al no tratar o subtratar el dolor del enfermo.

El manejo del dolor en el paciente traumatizado es un reto. La evaluación inicial y el tratamiento de lesiones que ponen en riesgo la vida o la extremidad tienen prioridad y el inicio de la analgesia frecuentemente se pospone hasta que el paciente está estable. El tratamiento de primera línea para el paciente traumatizado son los opiáceos en dosis bajas administrados de manera intravenosa. Son rápidos, efectivos y están indicados en el dolor severo. Sin embargo, existen múltiples razones por las que el paciente traumatizado recibe una analgesia insuficiente: los médicos oponen resistencia a administrar opiáceos por vía sistémica debido al temor de agravar la inestabilidad hemodinámica o la depresión respiratoria con pérdida de la vía aérea. Además, en los pacientes con traumatismo craneoencefálico les preocupa el enmascaramiento del cuadro clínico o producir delirio, en particular en los pacientes de edad avanzada, lo cual puede confundir el cuadro clínico del traumatismo craneoencefálico, además de que se necesitan evaluaciones frecuentes del paciente sin sedación y no se puede estimar si el paciente realmente se está deteriorando. ${ }^{16,17}$
Otra de las razones por las que los pacientes reciben oligoanalgesia es porque es difícil medir el dolor en trauma debido a que a menudo los pacientes están intubados y con ventilación mecánica asistida y no se puede establecer una comunicación. ${ }^{18}$

Con base en lo anterior, la analgesia multimodal y en particular la analgesia regional como la ablación por radiofrecuencia reducen de manera importante los requerimientos de opiáceos, acortando la estancia en la unidad de terapia intensiva, salas de emergencia, recuperación, y disminuyen los efectos adversos de estos medicamentos como: náusea, vómito, cefalea, depresión respiratoria, delirio, vasodilatación con hipotensión, constipación, prurito, inmunodepresión y el requerir de mayor número de personal para su vigilancia y monitorización.

En esta serie de casos fue evidente cómo la radiofrecuencia de los nervios intercostales redujo de manera importante el consumo de morfina en mg por 24 horas (70.8\%) al producir una analgesia de alta calidad que es específica para la pared torácica, y por lo tanto, sin efectos sistémicos.

En nuestros pacientes el dolor con la ablación de los nervios intercostales mediante radiofrecuencia disminuyó de un dolor severo 7-10 media de 7.5 puntos a la ventilación superficial en escala numérica del dolor, a un dolor de 2-4 con media de 2, siendo un dolor leve y atribuido a que también existen otras causas de dolor en el trauma de tórax independientes de las fracturas costales. Lo anterior representó un alivio aproximado de $70 \%$ en la mayoría de los casos al ser posible manejarlos sin opiáceos con otro tipo de analgésicos, lo que fue de gran ayuda para la unidad de terapia intensiva al poder extubar de manera más temprana a los enfermos por tener una mucho mejor mecánica respiratoria, logrando realizar una inspiración y espiración más profundas y por consiguiente, un mejor manejo de secreciones de la vía aérea y menos posibilidades de complicarse con atelectasia pulmonar y neumonía.

Los resultados observados en nuestro estudio están en concordancia con la literatura mundial al observar una reducción importante del consumo de morfina diaria. ${ }^{15,19}$ 


\section{CONCLUSIÓN}

El trauma es un problema de salud pública a nivel mundial y una de las principales causas de mortalidad en todos los grupos etarios. El dolor que produce particularmente el trauma contuso de tórax es intenso, por lo regular requiere de opiáceos y no es raro que por diversas razones se encuentre subtratado.

La ablación térmica de los nervios intercostales por radiofrecuencia en trauma contuso de tórax con fracturas costales múltiples y tórax inestable es útil y segura para el tratamiento de los pacientes. En esta serie de casos fue evidente cómo la radiofrecuencia de los nervios intercostales disminuyó de manera importante el consumo de morfina en $\mathrm{mg}$ por 24 horas $(70.8 \%)$ al producir una analgesia de alta calidad que es específica para la pared torácica y por lo tanto, sin efectos secundarios.

Esta técnica reduce el dolor en forma significativa y permite mejor ventilación con menor consumo de otros analgésicos.

La ablación térmica de los nervios intercostales puede perfilarse como un nuevo estándar de oro en el control del dolor en trauma torácico severo. Se requieren estudios más amplios con mayor número de pacientes para continuar validando estas conclusiones iniciales.

\section{REFERENCIAS}

1. Engel AJ. Utility of intercostal nerve conventional thermal radio frequency ablation in the injured worker after blunt trauma. Pain Physician. 2012; 15: E711-E718.

2. Fagevik-Olsén M, Slobo M, Klarin L, Caragounis EC, Pazooki D, Granhed H. Physical function and pain after surgical or conservative management of multiple rib fractures - a follow-up study. Scand J Trauma Resusc Emerg Med. 2016; 24: 128.

3. VanSonnenberg E, Shankar S, Morrison PR, Nair RT, Silverman SG, Jaklitsch MT, et al. Radiofrequency ablation of thoracic lesions: part 2, initial clinical experience--technical and multidisciplinary considerations in 30 patients. AJR Am J Roentgenol. 2005; 184: 381-390.

4. Samson PC, Fitzpatrick LJ. Intercostal nerve block: its rôle in the management of thoracic casualties. Cal West Med. 1945; 62: 254-256.

5. Joshi GP, Bonnet F, Shah R, Wilkinson RC, Camu F, Fischer B, et al. A systematic review of randomized trials evaluating regional techniques for postthoracotomy analgesia. Anesth Analg. 2008; 107: 1026-1040.

6. Cohen SP, Sireci A, Wu CL, Larkin TM, Williams KA, Hurley RW. Pulsed radiofrequency of the dorsal root ganglia is superior to pharmacotherapy or pulsed radiofrequency of the intercostal nerves in the treatment of chronic postsurgical thoracic pain. Pain Physician. 2006; 9: 227-235.

7. Bogduk N. Pulsed radiofrequency. Pain Med. 2006; 7: 396-407.

8. Chua NH, Vissers KC, Sluijter ME. Pulsed radiofrequency treatment in interventional pain management: mechanisms and potential indications-a review. Acta Neurochir (Wien). 2011; 153: 763-771.

9. Govind J, King W, Bailey B, Bogduk N. Radiofrequency neurotomy for the treatment of third occipital headache. J Neurol Neurosurg Psychiatry. 2003; 74: 88-93.

10. Bogduk N, Holmes S. Controlled zygapophysial joint blocks: the travesty of cost-effectiveness. Pain Med. 2000; 1: 24-34.

11. Kerr-Valentic MA, Arthur M, Mullins RJ, Pearson TE, Mayberry JC. Rib fracture pain and disability: can we do better? J Trauma. 2003; 54: 1058-1063; discussion 1063-1064.

12. Gekht G, Nottmeier EW, Lamer TJ. Painful medial branch neuroma treated with minimally invasive medial branch neurectomy. Pain Med. 2010; 11: 1179-1182.

13. Manchikanti L, Pampati V, Damron KS, McManus $\mathrm{CD}$, Jackson SD, Barnhill RC, et al. A randomized, prospective, double-blind, placebo-controlled evaluation of the effect of sedation on diagnostic validity of cervical facet joint pain. Pain Physician. 2004; 7: 301-309.

14. Atlas SJ, Tosteson TD, Blood EA, Skinner JS, Pransky GS, Weinstein JN. The impact of workers' compensation on outcomes of surgical and nonoperative therapy for patients with a lumbar disc herniation: SPORT. Spine (Phila Pa 1976). 2010; 35: 89-97.

15. Gadsden J, Warlick A. Regional anesthesia for the trauma patient: improving patient outcomes. Local Reg Anesth. 2015; 8: 45-55.

16. Cortés F, Buitrago F. Trauma de tórax. Rev Fac Med. 2000; 48: 35-44.

17. Martínez Casas I, Amador Marchante MA, Paduraru M, Fabregues Olea Al, Nolasco A, Medina JC. Thorax trauma severity score: is it reliable for patient's evaluation in a secondary level hospital? Bull Emerg Trauma. 2016; 4: 150-155.

18. Yeying G, Liyong Y, Yuebo C, Yu Z, Guangao Y, Weihu $M$, et al. Thoracic paravertebral block versus intravenous patient-controlled analgesia for pain treatment in patients with multiple rib fractures. J Int Med Res. 2017; 45: 2085-2091.

19. Kim YJ, Cho HM, Yoon CS, Lee CK, Lee TY, Seok JP. Thoracic epidural anesthesia and analgesia (TEA) in patients with rib fractures. Korean J Thorac Cardiovasc Surg. 2011; 44: 178-182. 
Consideraciones y responsabilidad ética: Privacidad de los datos. De acuerdo a los protocolos establecidos en el centro de trabajo de los autores, éstos declaran que han seguido los protocolos sobre la privacidad de datos de pacientes preservando su anonimato. El consentimiento informado del paciente referido en el artículo se encuentra en poder del autor. Financiamiento: No se recibió apoyo financiero para la realización de este trabajo.
Conflicto de intereses: Los autores declaran que no existe ningún conflicto de intereses en la realización del trabajo.

Correspondencia: Dr. Rafael Acuña Prats

E-mail: acunaprats@gmail.com 